Pacific Journal of Mathematics

LOCAL CONTROL AND FACTORIZATION OF THE FOCAL 


\title{
LOCAL CONTROL AND FACTORIZATION OF THE FOCAL SUBGROUP
}

\author{
DAVID FINKEL
}

There are a number of results that yield information about the structure of a finite group $G$, based on information about its local subgroups; that is, the normalizers of its $p$ subgroups. Alperin and Gorenstein defined a notion of control of transfer, fusion, or strong fusion in a group $G$ by a conjugacy functor $W$, or rather by $N(W(P))$, where $P$ is a Sylow $p$-subgroup of $G$, and proved that if $W$ controls transfer, fusion or strong fusion in every local subgroup of $G$, then $W$ controls, respectively, transfer, fusion or strong fusion in the entire group $G$.

This paper gives a definition of control by two functors, $W$ and $V$, or rather $N(W(P))$ and $C(V(P))$, and proves that when $W$ contains the center, and when $V$ is contained in the center and satisfies another condition, control in certain local subgroups implies control in the entire group $G$. In particular, the result on transfer says that $P \cap G^{\prime}$, the focal subgroup of $G$, can be factorized whenever their focal subgroups can be properly factorized. From theorems of Glauberman, Goldschmidt, and Thompson which give conditions under which a group can be factorized, it is possible to obtain results which say that the focal subgroup of $G$ can be factorized whenever certain local subgroups are $p$-solvable and satisfy the hypotheses of the factorization theorems.

1. Introduction. We use throughout the notation of [5]. In particular, $H$ is a subgroup of $G$ is denoted by $H \leqq G$, with $H<G$ reserved for proper subgroups. We fix $G$ a finite group, $p$ a prime dividing the order of $G$, and $P$ a Sylow $p$-subgroup of $G$.

Definition 1.1. ([2], p. 242) Let $\mathscr{H}$ be the set of all non-identity $p$-subgroups of $G$. A conjugacy functor $W$ on $\mathscr{H}$ is a mapping from $\mathscr{H}$ to $\mathscr{\mathscr { C }}$ satisfying, for each $H \in \mathscr{X}$

(a) $W(H) \leqq H$, and

(b) $W\left(H^{x}\right)=W(H)^{x}$ for all $x \in G$.

DEFINITION 1.2. A central functor $V$ on $\mathscr{H}$ is a conjugacy functor satisfying, for any $H, K \in \mathscr{C}$

(a) $V(H) \leqq Z(H)$

(b) if $Z(H) \leqq Z(K)$, then $V(H) \leqq V(K)$.

REMARK 1.3. Examples of conjugacy functors include the identity 
map $W(H)=H$, and the Thompson subgroup, $J(H)$, which is the subgroup of $H$ generated by all Abelian subgroups of $H$ for which the minimal number of generators is maximal. Examples of central functors include the center $Z(H)$ and the functor $V(H)=\tilde{O}^{n}(Z(H))$, the subgroup of $H$ generated by all $p^{n}$ th powers of elements of $Z(H)$.

We now define a special notion of fusion.

Definition 1.4. Let $A$ and $B$ be subsets of $G$, and $K_{1}$ and $K$ subsets of $G$ with $K_{1} \subseteq K$, and $g \in\langle A, B\rangle$ such that $K_{1}^{g} \subseteq K$. We say $g$ takes $K_{1}$ to $K_{1}^{g}$ in $K$ via $A$ and $B$ if we can write $g=g_{1} g_{2} \cdots g_{n}$ with $g_{i} \in A$ or $B$, and $K_{1}^{g_{1} \cdots g_{i}} \subseteq K$ for all $i=1,2, \cdots, n$. That is, we require that $K_{1}$ be conjugate to $K_{1}^{g}$ by a series of conjugations so that each intermediate step lies in $K$.

REMARK 1.5. (a) If $g$ takes $K_{1}$ to $K_{1}^{g}$ in $K$ via $A$ and $B$, and if $K \subseteq L ; A \subseteq C, B \subseteq D$ and if $K_{2} \subseteq K_{1}$ then $g$ takes $K_{2}$ to $K_{2}^{g}$ in $L$ via $C$ and $D$.

(b) If $g_{1}$ takes $K_{1}$ to $K_{2}$ in $K$ via $A$ and $B$, and $g_{2}$ takes $K_{2}$ to $K_{3}$ in $K$ via $A$ and $B$ then the product $g_{1} g_{2}$ takes $K_{1}$ to $K_{3}$ in $K$ via $A$ and $B$.

Using this notion of fusion, we can now define local control, and state our main theorem.

DEFINITION 1.6. Let $W$ be a conjugacy functor on $\mathscr{H}$, and $V$ a central functor on $\mathscr{H}$, and $L$ a subgroup of $G$ with order divisible by p. We say, respectively, that $W$ with $V$ controls

(1) transfer in $L$

(2) fusion in $L$

(3) strong fusion in $L$

provided there exists a Sylow $p$-subgroup $Q$ of $L$ such that

(1) $Q \cap L^{\prime}=\left(Q \cap N_{L}(W(Q))^{\prime}\right) \cdot\left(Q \cap C_{L}(V(Q))^{\prime}\right)$

(2) Whenever $X$ and $Y$ are subsets of $Q$ conjugate in $L$, then they are conjugate by an element of $\left\langle N_{L}(W(Q)), C_{L}(V(Q))\right\rangle$ which takes $X$ to $Y$ in $Q$.

(3) Whenever $X$ and $Y$ are subsets of $Q$, with $Y=X^{a}$ for some $a \in L$, then $a=c b$, with $c \in C_{L}(X), b \in\left\langle N_{L}(W(Q)), C_{L}(V(Q))\right\rangle$, and $b$ takes $X$ to $Y$ in $Q$.

DEFINITION 1.7. A local subgroup of $G$ is a subgroup of the form $L=N(H)$, for some $H \in \mathscr{H}$. Define $\mathscr{L}$ as the set of those local subgroups satisfying $L=N(H)$, with $Z(P) \leqq H \leqq P$.

We now state our main theorem, relating local and global control by two functors, $W$ and $V$. Note that we require $Z(H) \leqq W(H)$ and $V(H) \leqq Z(H)$. 
THEOREM 1.8. Let $W$ be a conjugacy functor on $\mathscr{H}$ satisfying $Z(H) \leqq W(H)$ for all $H \in \mathscr{H}$, and let $V$ be a central functor on $\mathscr{H}$. Assume that $W$ with $V$ controls

(1) transfer,

(2) fusion, or

(3) strong fusion

in all local subgroups $L \in \mathscr{L}$. Then $W$ with $V$ controls, respectively, transfer, fusion, or strong fusion in $G$.

We use Theorem 1.8 (1), on control of transfer, to obtain factorizations of the focal subgroup, $P \cap G^{\prime}$, when the local subgroups in $\mathscr{L}$ are all $p$-solvable and satisfy certain other conditions. This is discussed in $\S 6$.

2. Assumed results. We need a number of concepts and results from Alperin [1] and Alperin and Gorenstein [2]. Throughout, as in the previous chapter, $G$ is a finite group, and $P$ is a Sylow $p$-subgroup of $G$. Also, $W$ is a conjugacy functor on $\mathscr{H}$, as in Definition 1.1, and in addition, satisfies $W(H) \geqq Z(H)$ for all $H \in \mathscr{H}$.

Definition 2.1. ([1], p. 236) A family is a collection of pairs $(H, T)$ where $H$ is a subgroup of $P$ and $T$ is a subset of $N(H)$.

Definition 2.2. ([1], p. 237) A family $F$ is called a conjugation family provided that whenever $A$ and $B$ are subsets of $P$ and $B=A^{g}$ for some $g \in G$, then there exist elements $\left(H_{1}, T_{1}\right),\left(H_{2}, T_{2}\right), \cdots,\left(H_{n}, T_{n}\right)$ of $F$, and elements $x_{1}, x_{2}, \cdots, x_{n}$, and $y$ of $G$ satisfying

(a) $g=x_{1} \cdots x_{n} y$

(b) $x_{i} \in T_{i}$, for $i=1,2, \cdots, n$, and $y \in N(P)$

(c) $A \leqq H_{1}, A^{x_{1} \cdots x_{i}} \leqq H_{i+1}$, for $i=1, \cdots, n+1$.

We now define a particular conjugation family which we use for the remainder of the paper.

Definition 2.3. ([1], p. 225) If $Q$ and $R$ are Sylow $p$-subgroups of $G$, then we say $Q \cap R$ is a tame intersection provided that $N_{Q}(Q \cap R)$ and $N_{R}(Q \cap R)$ are Sylow $p$-subgroups of $N(Q \cap R)$.

Definition 2.4. ([2], p. 245) If $H$ is a subgroup of $P$, we set $W_{1}(H)=H, M_{1}(H)=N_{P}(H)$, and $N_{1}(H)=N(H)$, and define recursively $W_{i+1}(H)=W\left(M_{i}(H)\right), M_{i+1}(H)=N_{P}\left(W_{i+1}(H)\right)$, and $N_{i+1}(H)=N\left(W_{i+1}(H)\right)$.

REMARK 2.5. As noted in [2], page 245, the sequence $M_{i}(H)$ is strictly increasing until it stabilizes at $P$. Also, if $M_{i}(H)=P$, then $W_{i+1}(H)=W(P)$, so that the sequence $W_{i}(H)$, though not necessarily 
increasing, stabilizes at $W(P)$.

DeFinition 2.6. ([2], p. 245) If $H$ is a subgroup of $P$, we say that $H$ is well-placed in $P$ with respect to $W$ if $M_{i}(H)$ is a Sylow $p$-subgroup $N_{i}(H)$, for all $i$.

Proposition 2.7. ([2], p. 252) If $H$ is a subgroup of $P$, then some conjugate of $H$ is well-placed in $P$.

Definition 2.8. ([2], p. 246) We define the family $F$ to consist of all pairs $(H, T)$ such that there exists a Sylow $p$-subgroup $S$ of $G$ so that $H=P \cap S$ is a well-placed tame intersection, and

(a) $T=C(H)$ if $C_{P}(P \cap S) \not \equiv P \cap S$

(b) $T=N(H)$ if $C_{P}(P \cap S) \leqq P \cap S$.

REMARK 2.9. Note that $(P, N(P)) \in F$. This fact eliminates the need for special mention of $y \in N(P)$ in Definition 2.2.

Proposition 2.10. $F$ is a conjugation family.

Proof. Section 2 of [2] is devoted to a proof of this fact.

In the next section, we use the following result, which applies to all conjugation families.

Proposition 2.11. ([1], p. 238) If $P^{*}$ is a subgroup of $P$, and if $[P, N(P)] \leqq P^{*}<P \cap G^{\prime}$, then there is an element $(H, T)$ of $F$ such that $[H, T] \geqq P^{*}$.

3. Control of transfer. We now turn to the proof of Theorem 1.8 (1), on local control of transfer.

Lemma 3.1. Let $P^{*}$ be a subgroup of $G$ satisfying $P \cap(N(W(P)))^{\prime} \leqq$ $P^{*}<P \cap G^{\prime}$. Then there exists a non-identity subgroup $K$ of $P$ such that

(a) $Z(P) \leqq K$

(b) $P \cap L$ is a Sylow p-subgroup of $L=N(K)$

(c) $P \cap L^{\prime} \not P^{*}$, and

(d) $P \cap M^{\prime} \leqq P^{*}$ where $M=N(W(P \cap L))$.

Proof. By the definition of conjugacy functor, if $x \in N(P)$, then $W(P)=W\left(P^{x}\right)=W(P)^{x}$, so that $N(P) \leqq N(W(P))$. Thus, $P \cap N(P)^{\prime} \leqq$ $P \cap N(W(P))^{\prime}$, and the hypotheses of this lemma imply that $P \cap N(P)^{\prime} \leqq$ $P^{*}$. Thus all the hypotheses of Proposition 2.11 are fulfilled, and we conclude that there exists $(H, T) \in F$ such that $[H, T] \not P^{*}$. 
Now, if $C_{P}(H) \nsubseteq H$, then by the definition of $F$, Definition 2.8, we have $T=C(H)$, so $[H, T] \leqq P^{*}$, contrary to our choice of $(H, T)$. Thus, we must have $C_{P}(H) \leqq H$ and $T=N(H)$.

Thus, $Z(P) \leqq H$, so (a) is satisfied for $K=H$. Furthermore, as $[H, N(H)] \leqq N(H)^{\prime}$, and $[H, N(H)] \leqq P$, we have $[H, N(H)] \leqq P \cap N(H)^{\prime}$. Thus, $[H, N(H)] \not P^{*}$ implies $P \cap N(H)^{\prime} \not \equiv P^{*}$, as required in (c). Also, $H$ is, by choice, a tame intersection in $P$, so $N_{P}(H)=P \cap N(H)$ is a Sylow $p$-subgroup of $N(H)$, as required in (b). Thus, (a), (b), and (c) hold for $K=H$.

Define $W_{i}=W_{i}(H)$, so that $W_{1}=H$. We shall find an $m$ so that $W_{m}$ satisfies (d) as well as (a), (b), and (c).

First, we show that $Z(P) \leqq W_{i}$ for all $i$. For $i=1$, we have seen that $Z(P) \leqq W_{1}=H$. For $i>1$, as $W_{i-1} \leqq P$, we have $Z(P) \leqq$ $N\left(W_{i-1}\right) \cap P$, so $Z(P) \leqq Z\left(N\left(W_{i}\right) \cap P\right)$. By our choice of $W$, we have $Z(L) \leqq W(L)$ for all $L \in \mathscr{C}$, so

$$
Z(P) \leqq Z\left(N\left(W_{i-1}\right) \cap P\right) \leqq W\left(N\left(W_{i-1}\right) \cap P\right)=W_{i} \text {. }
$$

Thus, $Z(P) \leqq W_{i}$ for all $i$, so each $W_{i}$ satisfies (a).

As $H$ is well-placed, each $M_{i}(H)$ is a Sylow $p$-subgroup of $N_{i}(H)$. That is, $N_{P}\left(W_{i}(H)\right)=P \cap\left(N\left(W_{i}(H)\right)\right.$ is a Sylow $p$-subgroup of $N\left(W_{i}(H)\right)$. Thus, (b) is also satisfied for each $W_{i}$.

Now, as noted in Remark 2.5, $W_{i}=W(P)$ for all $i$ sufficiently large, and, as we have $P \cap N(W(P))^{\prime} \leqq P^{*}$, (c) does not hold for $W_{i}$ with $i$ sufficiently large. However, (c) does hold for $i=1$. Thus, we can choose $m$ so that (c) holds $W_{m}$, but not for $W_{m+1}$. But $W_{m+1}=$ $W\left(P \cap N\left(W_{m}\right)\right)$, so $P \cap N\left(W_{m+1}\right)^{\prime} \leqq P^{*}$, as (c) fails for $W_{m+1}$, and so $P \cap N\left(W\left(P \cap N\left(W_{m}\right)\right)\right)^{\prime} \leqq P^{*}$. But this last inequality states that contition (d) holds for $W_{m}$.

Thus, setting $K=W_{m}$ completes the proof of the lemma.

We now complete the proof of the theorem on control of transfer.

Proof of Theorem 1.8 (1). Assume that the conclusion of the theorem is false. Then we have

$$
P \cap N(W(P))^{\prime} \cdot P \cap C(V(P))^{\prime}<P \cap G^{\prime} .
$$

Call the group on the left $P^{*}$. Then, as $P^{*}<P \cap G^{\prime}$, we may apply Lemma 3.1, and find subgroups $K, L$, and $M$ as defined in the lemma.

As $L$ is a local subgroup, the hypotheses of our theorem imply that $W$ with $V$ controls transfer in $L$, so there exists $Q$, a Sylow $p$-subgroup of $L$, satisfying

$$
Q \cap L^{\prime}=\left[Q \cap C_{L}(V(Q))^{\prime}\right] \cdot\left[Q \cap N_{L}(W(Q))^{\prime}\right] .
$$

By conjugacy, this statement holds for all Sylow $p$-subgroups of 
$L$, so we may take $Q=P \cap L$, which is a Sylow $p$-subgroup of $L$ by (b) of the lemma. Thus, using the fact that $M=N(W(P \cap L))$, the above equation becomes

$$
(P \cap L) \cap L^{\prime}=\left[(P \cap L) \cap C_{L}(V(P \cap L))^{\prime}\right] \cdot\left[(P \cap L) \cap(M \cap L)^{\prime}\right]
$$

which yields

$$
P \cap L^{\prime}=\left[P \cap C_{L}(V(P \cap L))^{\prime}\right]\left[P \cap(M \cap L)^{\prime}\right]
$$

From (d) of the lemma, $P \cap M^{\prime} \leqq P^{*}$, so certainly $P \cap(M \cap L)^{\prime} \leqq$ $P^{*}$. Also, from (a) of the lemma $Z(P) \leqq K$, and $K \leqq L$, so $Z(P) \leqq$ $P \cap L \leqq P$, which implies that $Z(P) \leqq Z(P \cap L)$, and so $C(V(P \cap L)) \leqq$ $C(V(P))$. Thus, $P \cap C_{L}(V(P \cap L))^{\prime} \leqq P \cap C(V(P))^{\prime} \leqq P^{*}$ by the definition of $P^{*}$. Hence, from (3.2), we have

$$
P \cap L^{\prime}=\left[P \cap C_{L}(V(P \cap L))^{\prime}\right] \cdot\left[P \cap(M \cap L)^{\prime}\right] \leqq P^{*},
$$

contrary to (c) of the lemma.

This contradiction completes the proof of Theorem 1.8 (1).

4. Control of fusion. Before proving Theorem 1.8 (2), we need first the following lemma. Recall that, if $g \in\langle A, B\rangle$ takes $X$ to $Y$ in $P$, then Definition 1.4 requires that the intermediate steps lie in $P$.

Lemma 4.1. Assume the hypotheses of Theorem 1.8 (2). Assume further that we have $Q$, a Sylow p-subgroup of $G$, so that $P \cap Q$ is a well-placed, tame intersection and $C_{P}(P \cap Q) \leqq P \cap Q$, and that we have $X$ and $Y$ subsets of $P \cap Q$, satisfying $X^{g}=Y$ for some $g \in N(P \cap Q)$. Then $X$ and $Y$ are conjugate by an element of $\langle N(W(P)), C(V(P))\rangle$ which takes $X$ to $Y$ in $P$.

Proof. Let $W_{i}=W_{i}(P \cap Q), M_{i}=M_{i}(P \cap Q)$, and $N_{i}=N_{i}(P \cap Q)$, as defined in Definition 2.4, and let $C_{i}=\left\langle N_{i}, C(V(P))\right\rangle$. We show by induction on $i$ that there exists $c_{i} \in C_{i}$ satisfying $Y=X^{c_{i}}$ and $c_{i}$ takes $X$ to $Y$ in $M_{i}$ via $N_{i}$ and $C(V(P))$. Since, by Remark 2.5, $N_{i}=$ $N(W(P))$ and $M_{i}=P$ for $i$ sufficiently large, this will suffice.

For $i=1$, we have $N_{1}=N(P \cap Q)$, and $N_{1} \leqq C_{1}$. Thus, $g \in C_{1}$, and $X^{g}=Y$ and $g$ takes $X$ to $Y$ in $M_{1}$ via $N_{1}$ and $C(V(P))$, as $g \in N_{1}$ and $X, Y \leqq P \cap Q \leqq M_{1}$. Thus, $c_{1}=g$.

Assume, by induction, that $X^{c_{i}}=Y$ for some $c_{i} \in C_{i}$, and $c_{i}$ takes $X$ to $Y$ in $M_{i}$. That is, we have $c_{i}=a_{1} a_{2} \cdots a_{n}$, with $a_{k} \in N_{i}$ or $a_{k} \in C(V(P))$, for $k=1,2, \cdots, n$, and, if we call $X_{k}=X^{a_{1} \cdots a_{k}}$, then we have $X=X_{0}, Y=X_{n}$, and $X_{k} \leqq M_{i}$ for $k=1, \cdots, n$. 
It suffices to show that, for each $k=1, \cdots, n$, we have $X_{k-1}$ and ? $X_{k}$ conjugate by an element $b_{k} \in C_{i+1}$ which takes $X_{k-1}$ to $X_{k}$ in $M_{i+1}$ via $N_{i+1}$ and $C(V(P))$. If we show this, then the product $b_{1} \cdots b_{n}$ takes $X$ to $Y$ in $M_{i+1}$, as required.

If $a_{k} \in C(V(P))$, then $X_{k-1}$ and $X_{k}$ are conjugate by an element of $C_{i+1}$, namely $a_{k}$ itself, and, as $M_{i} \leqq M_{i+1}$, we see that $a_{k}$ takes $X_{k-1}$ to $X_{k}$ in $M_{i+1}$ as required. In this case, let $b_{k}=a_{k}$.

So we may assume $a_{k} \in N_{i}$. We now claim that $W$ with $V$ controls fusion in $N_{i}$. To establish this, we need only prove that $Z(P) \leqq$ $W_{i}$, since $N_{i}=N\left(W_{i}\right)$ and we are assuming that $W$ with $V$ controls fusion in every local subgroup of the form $L=N(H)$ with $Z(P) \leqq$ $H \leqq P$.

Since $C_{P}(P \cap Q) \leqq P \cap Q$, by hypothesis of the lemma, we have $Z(P) \leqq P \cap Q=W_{1}$. Assume now, by induction, that $Z(P) \leqq W_{j}$. Then $Z\left(M_{j}\right) \leqq W\left(M_{j}\right)=W_{j+1}$, by hypothesis on $W$. But $Z(P) \leqq W_{j} \leqq$ $M_{j} \leqq P$, so $Z(P) \leqq Z\left(M_{j}\right)$ and $Z(P) \leqq W_{j+1}$. Thus, for all $i, Z(P) \leqq$ $W_{i}$, and $W$ with $V$ controls fusion in $N_{i}$ as claimed.

Thus, as we have $X_{k-1}$ and $X_{k}$, subsets of $M_{i}$, conjugate by $a_{k} \in N_{i}$ and $M_{i}$ is a Sylow $p$-subgroup of $N_{i}$, as $H$ is well-placed, we conclude that $X_{k-1}$ and $X_{k}$ are conjugate by some element

$$
b_{k} \in\left\langle N\left(W\left(M_{i}\right)\right), C\left(V\left(M_{i}\right)\right)\right\rangle
$$

which takes $X_{k-1}$ to $X_{k}$ in $M_{i}$. Also, $Z(P) \leqq P \cap N_{i}=M_{i}$, so $Z(P) \leqq$ $Z\left(M_{i}\right)$ and $V(P) \leqq V\left(M_{i}\right)$. Thus $C\left(V\left(M_{i}\right)\right) \leqq C(V(P))$, and

$$
b_{k} \in\left\langle N\left(W\left(M_{i}\right)\right), C(V(P))\right\rangle=C_{i+1},
$$

and since $M_{i} \leqq M_{i+1}$, it follows that $b_{k}$ takes $X_{k-1}$ to $X_{k}$ in $M_{i+1}$, as required. This completes the induction on $i$ and the proof of the lemma.

We now complete the proof of Theorem 1.8 (2).

Proof of Theorem 1.8 (2). Let $X$ and $Y$ be subsets of $P$ conjugate in $G$. We wish to show that $X$ and $Y$ are conjugate by an element of $\langle N(W(P)), C(V(P))\rangle$ which takes $X$ to $Y$ in $P$ via $N(W(P))$ and $C(V(P))$.

Using the conjugation family $F$ of Definition 2.8, we know there exist $X_{0}, X_{1}, \cdots, X_{n}$, subsets of $P$, and $\left(H_{i}, T_{i}\right) \in F$ such that $X=X_{0}$, $X_{n}=Y$, and $X_{i-1}$ and $X_{i}$ are contained in $H_{i}$ and conjugate by an element of $T_{i}$.

To prove our theorem, it suffices to prove that for each $i=$ $1, \cdots, n, X_{i-1}$ and $X_{i}$ are conjugate by an element $g_{i} \in\langle N(W(P)), C(V(P))\rangle$ taking $X_{i-1}$ to $X_{i}$ in $P$, for then their product $g_{1} g_{2} \cdots g_{n} \in\langle N(W(P))$, 
$C(V(P))\rangle$ takes $X$ to $Y$ in $P$ via $N(W(P))$ and $C(V(P))$.

If $C_{P}\left(H_{i}\right) \not H_{i}$, then $T_{i}=C\left(H_{i}\right)$, by definition of $F$, so $X_{i-1}=X_{i}$, and the assertion holds. In this case, set $g_{i}=1$.

If, on the other hand, $C_{P}\left(H_{i}\right) \leqq H_{i}$, we may use Lemma 4.1 , for $H_{i}$ is a well-placed, tame intersection of $P$ with some other Sylow $p$-subgroup of $G$. Thus, we can apply Lemma 4.1 to $X_{i-1}$ and $X_{i}$, and conclude that $X_{i-1}$ and $X_{i}$ are conjugate by an element

$$
g_{i} \in\langle N(W(P)), C(V(P))\rangle
$$

taking $X_{i-1}$ to $X_{i}$ in $P$.

Thus, $g_{1} g_{2} \cdots g_{n}$ takes $X$ to $Y$ in $P$ as required, and the proof of Theorem $1.8(2)$ is complete.

5. Control of strong fusion. To prove Theorem 1.8 (3), on control of strong fusion, we need first the following theorem.

Theorem 5.1. Assume the hypotheses of Theorem 1.8 (3). Let $X$ be a subgroup of $P$, and $g \in N(X)$. Then we can write $g=a b$, where $a \in C(X), b \in\langle N(W(P)), C(V(P))\rangle$ and $b$ takes $X$ to $X$ in $P$.

REMARK 5.2. Proving Theorem 5.1 will suffice to prove Theorem 1.8 (3). For if $X$ and $Y$ are subsets of $P$, with $X^{g}=Y$ for some $g \in G$, then, by Theorem $1.8(2)$, there exists $h \in\langle N(W(P)), C(V(P))\rangle$ such that $X^{h}=Y$ and $h$ takes $X$ to $Y$ in $P$. Thus, $g h^{-1} \in N(X)$. We may apply the theorem above to the subgroup $\langle X\rangle$, and as $g h^{-1} \in$ $N(\langle X\rangle)$, we have $g h^{-1}=a b$ for some $a \in C(\langle X\rangle)=C(X)$ and $b \in$ $\langle N(W(P)), C(V(P))\rangle$ and $b$ takes $X$ to $Y$ in $P$. Then $g=a b h$, where $a \in C(X), b h \in\langle N(W(P)), C(V(P))\rangle$ and $b h$ takes $X$ to $Y$ in $P$, as required to prove the theorem on control of strong fusion.

To prove Theorem 5.1, we need the following lemmas. For the remainder of this section assume the hypotheses of Theorem 1.8 (3), namely that $W$ with $V$ controls strong fusion in all local subgroups of the form $L=N(H)$ with $Z(P) \leqq H \leqq P$.

Lemma 5.3. If $H$ is well-placed in $P$, and $Z(P) \leqq H$, and if $g \in N(H)$, then $g \in\langle N(W(P)), C(V(P))\rangle$, and $g$ takes $H$ to $H$ in $P$.

Proof. Let $W_{i}=W_{i}(H), M_{i}=M_{i}, N_{i}=N_{i}(H)$, and

$$
C_{i}=\left\langle N_{i}, C(V(P))\right\rangle,
$$

as before. For $g \in N(H)$, we shall show by induction on $i$ that $g \in C_{i}$ and that $g$ takes $H$ to $H$ in $M_{i}$, via $N_{i}$ and $C(V(P))$. As before, this will suffice to prove the lemma.

For $i=1$, we have $g \in N(H)=N_{1}(H)$, so that $g \in C_{1}$. Further- 
more, $H \leqq N_{P}(H)=M_{1}(H)$, so that $g$ takes $H$ to $H$ in $M_{1}$ as required.

Assume now, by induction, that $g \in C_{i}$ and that $g$ takes $H$ to $H$ in $M_{i}$. Thus, $g=b_{1} \cdots b_{n}, b_{k} \in N_{i}$ or $b_{k} \in C(V(P))$, and $H^{b_{1} \cdots b_{k}} \leqq M_{i}$ for $k=1,2, \cdots, n$. Define $H_{0}=H$, and $H_{k}=H^{b_{1} \cdots b_{k}}$, for $k=1, \cdots, n$, so that $H_{n}=H$.

We now show, by induction on $k$, that $b_{k}$ takes $H_{k-1}$ to $H_{k}$ in $M_{i+1}$ via $N_{i+1}$ and $C(V(P))$. This will imply that $g=b_{1} \cdots b_{k}$ takes $H=H_{0}$ to $H=H_{n}$ in $M_{i+1}$ via $N_{i+1}$ and $C(V(P))$, which will complete the induction on $i$ and the proof of the lemma.

Assume $k=1$. If $b_{1} \in C(V(P))$, then $b_{1} \in C_{1}$. As $M_{i} \leqq M_{i+1}$, we have $b_{1}$ taking $H_{0}$ to $H_{1}$ in $M_{i+1}$ via $N_{i}$ and $C(V(P))$ as required.

Thus, we may assume that $b_{1} \in N_{i}$.

We now show that $W$ with $V$ controls strong fusion in $N_{i}$. By the hypotheses of the lemma, we need only show that $Z(P) \leqq W_{i}$, as $N_{i}=N\left(W_{i}\right)$. By hypothesis, $Z(P) \leqq H=W_{1}$. Assume now, by induction, that $Z(P) \leqq W_{j}$. Since $Z\left(M_{j}\right) \leqq W\left(M_{j}\right)$ by hypothesis on $W$, and $W_{j+1}=W\left(M_{j}\right)$, we have $Z\left(M_{j}\right) \leqq W_{j+1}$. As $Z(P) \leqq M_{j} \leqq P$, we have $Z(P) \leqq Z\left(M_{j}\right)$, so $Z(P) \leqq W_{j+1}$, completing the induction on $j$. Thus, $W$ with $V$ controls strong fusion in $N_{i}$ for all $i$.

Now $H_{0}^{b_{1}}=H_{1}$, where $H_{0}$ and $H_{1}$ are subsets of $M_{i}$, a Sylow $p$ subgroup of $N_{i}$, and so by control of strong fusion, we conclude that $b_{1}=c_{1} d_{1}$, where $c_{1} \in C(H), d_{1} \in\left\langle N\left(W\left(M_{i}\right)\right), C\left(V\left(M_{i}\right)\right)\right\rangle$, and $d_{1}$ takes $H_{0}$ to $H_{1}$ in $M_{i}$. Now since $V(P) \leqq Z(P) \leqq H$ by hypothesis, $C(H) \leqq$ $C(Z(P)) \leqq C(V(P))$, so $c_{1} \in C(V(P))$. Also, as we have seen, $Z(P) \leqq$ $M_{i} \leqq P$, so $Z(P) \leqq Z\left(M_{i}\right)$, and $C\left(V\left(M_{i}\right)\right) \leqq C(V(P))$, which implies that $d_{1} \in\left\langle N\left(W\left(M_{i}\right)\right), C(V(P))\right\rangle=C_{i+1}$. Furthermore, we have $M_{i} \leqq M_{i+1}$, and so $b_{1}=c_{1} d_{1} \in C_{i+1}$, and takes $H_{0}$ to $H_{1}$ in $M_{i+1}$ via $N_{i+1}$ and $C(V(P))$, as required, completing the case $k=1$.

We now consider the case $k>1$. We assume, by induction, that for all $r<k$, the result is true for $b_{r}$; that is that $b_{r}$ takes $H_{r-1}$ to $H_{r}$ in $M_{i+1}$ via $N_{i+1}$ and $C(V(P))$.

If $b_{k} \in C(V(P))$, we are done, as in the case $k=1$, for then $b_{k} \in$ $C_{i+1}$ immediately.

So we may assume that $b_{k} \in N_{i}$. As we have seen above, $W$ with $V$ controls strong fusion in $N_{i}$, and so $b_{k}=c_{k} d_{k}$ with $c_{k} \in C\left(H_{k-1}\right)$, $d_{k} \in C_{i+1}$, and $d_{k}$ takes $H_{k-1}$ to $H_{k}$ in $M_{i+1}$ via $N_{i+1}$ and $C(V(P))$. Now $c_{k} \in C\left(H_{k-1}\right)$, and $C\left(H_{k-1}\right)=C\left(H^{b_{1} b_{2} \cdots b_{k-1}}\right)=C(H)^{b_{1} b_{2} \cdots b_{k-1}}$. Thus, we have, for some $c \in C(H)$

$$
c_{k}=b_{k-1}^{-1} \cdots b_{1}^{-1} c b_{1} \cdots b_{k-1} .
$$

By our induction hypothesis on $k$, each $b_{r}$, for $r=1, \cdots, k-1$ takes $H_{r-1}$ to $H_{r}$ in $M_{i+1}$. Thus, each $b_{r}^{-1}$ takes $H_{r}$ to $H_{r-1}$ in $M_{i+1}$ via $N_{i+1}$ and $C(V(P))$. Furthermore, as $V(P) \leqq Z(P) \leqq H$, we have $C(H) \leqq C(V(P))$, so $c \in C(H)$ also takes $H$ to $H$ in $M_{i+1}$ via $N_{i+1}$ and 


\section{$C(V(P))$.}

We can now calculate the effect of $c_{k}$ on $H_{k-1}$ by examining the series of elements on the right-hand side of equation 5.4, and we find that $c_{k}$ takes $H_{k-1}$ to $H_{k-1}$ in $M_{i+1}$ via $N_{i+1}$ and $C(V(P))$.

Thus, $b_{k}=c_{k} d_{k}$ takes $H_{k-1}$ to $H_{k}$ in $M_{i+1}$ via $N_{i+1}$ and $C(V(P))$, completing the induction on $k$ and the proof of the lemma.

LEMma 5.5. Let $P \cap Q$ be a well-placed, tame intersection, with $C_{P}(P \cap Q) \leqq P \cap Q$, for $Q$ some Sylow p-subgroup of $G$. Let $H$ be a subgroup of $P \cap Q$, let $x \in N(P \cap Q)$, and suppose that for any $g \in N(H)$, we can write $g=a b$, where $a \in C(H), b \in\langle N(W(P)), C(V(P))\rangle$, and $b$ takes $H$ to $H$ in $P$. Then for any $h \in N\left(H^{x}\right)$, we can write $h=c d$, with $c \in C\left(H^{x}\right)$ and $d \in\langle N(W(P)), C(V(P))\rangle$ and $d$ takes $H^{x}$ to $H^{x}$ in $P$.

REMARK 5.6. This lemma shows that if Theorem 5.1 holds for such a subgroup $H$, then it holds for any conjugate of $H$ by an element of $N(P \cap Q)$.

Proof of lemma. Let $h \in N\left(H^{x}\right)$. Then $h^{x^{-1}} \in N(H)$, and so $h^{x^{-1}}=$ $a b$, where $a \in C(H), b \in\langle N(W(P)), C(V(P))\rangle$ and $b$ takes $H$ to $H$ in $P$.

Since $C_{P}(P \cap Q) \leqq P \cap Q$, we have $Z(P) \leqq P \cap Q$. Thus, the hypotheses of Lemma 5.3 are satisfied, with $P \cap Q$ in the place of $H$, and so, as $x \in N(P \cap Q)$, we have $x \in\langle N(W(P)), C(V(P))\rangle$ and $x$ takes $P \cap Q$ to $P \cap Q$ in $P$. Since $H$ and $H^{x}$ are subsets of $P \cap Q$, the subset property noted in Remark 1.5 shows that $x$ takes $H$ to $H^{x}$ in $P$. Similarly, $x^{-1}$ takes $H^{x}$ to $H$ in $P$.

Now $h^{x^{-1}}=a b$, so $h=\left(a^{x}\right)\left(b^{x}\right)$. As $a \in C(H), a^{x} C\left(H^{x}\right)$, as required. Furthermore, $b^{x} \in\langle N(W(P)), C(V(P))\rangle$, and as we have seen that $x^{-1}$ takes $H^{x}$ to $H$ in $P$, that $b$ takes $H$ to $H$ in $P$, and that $x$ takes $H$ to $H^{x}$ in $P$, we can see that $b^{x}$ takes $H^{x}$ to $H^{x}$ in $P$, by the transitivity property of Remark 1.5.

Thus, $h=\left(a^{x}\right)\left(b^{x}\right)$ is the required factorization of $h$, and the proof is complete.

LEMma 5.7. Let $Y$ be a well-placed subgroup of $P$ with $Z(P) \$ Y$, and assume that Theorem 5.1 holds for every subgroup $V$ of $P$ such that $Y<V$. Then Theorem 5.1 holds for $Y$.

Proof. Since $Z(P) \nsubseteq Y$, it follows that $C_{P}(Y) \$ Y$. Hence, $Y<$ $Y \cdot C_{P}(Y)$. Call $Y^{*}=Y \cdot C_{P}(Y)$, and note that, by hypothesis, the theorem holds for $Y^{*}$.

Now $Y$ is well-placed in $P$, so $N_{P}(Y)$ is a Sylow $p$-subgroup of $N(Y)$. Also, $Y \cdot C(Y)$ is normal in $N(Y)$, so $N_{P}(Y) \cap Y \cdot C(Y)$ is a Sylow $p$-subgroup of $Y \cdot C(Y)$. But $N_{P}(Y) \cap Y \cdot C(Y)=N(Y) \cap P \cap$ 
$Y \cdot C(Y)=P \cap Y \cdot C(Y)$, as $Y \cdot C(Y) \leqq N(Y)$. Furthermore, $P \cap$ $Y \cdot C(Y)=Y \cdot C_{P}(Y)=Y^{*}$ by the Dedekind identity. Thus, $Y^{*}$ is a Sylow $p$-subgroup of $Y \cdot C(Y)$, which is a normal subgroup of $N(Y)$. So we may apply the Frattini argument, and conclude $N(Y) \leqq$ $Y \cdot C(Y) \cdot N\left(Y^{*}\right)$.

But, as $Y \leqq Y^{*}$, certainly $Y \leqq N\left(Y^{*}\right)$, so the above relation may be rewritten as $N(Y) \leqq C(Y) \cdot N\left(Y^{*}\right)$.

Thus, if $g \in N(Y)$, we can write $g=c \cdot n$, for some $c \in C(Y)$, $n \in N\left(Y^{*}\right)$. Now Theorem 5.1 holds for $Y^{*}$, so $n=a b$, for some $a \in C\left(Y^{*}\right), b \in\langle N(W(P)), C(V(P))\rangle$, and $b$ takes $Y^{*}$ to $Y^{*}$ in $P$.

Now $Z(P) \leqq Y^{*}$, since $C_{P}(Y) \leqq Y^{*}$, so $C\left(Y^{*}\right) \leqq C(Z(P)) \leqq C(V(P))$, and so $a \in\langle N(W(P)), C(V(P))\rangle$, and takes $Y^{*}$ to $Y^{*}$ in $P$. But $n \in N(Y)$, and thus the subset property of Remark 1.5 shows that $n$ actually takes $Y$ to $Y$ in $P$.

Thus, $g=c \cdot n$, with $c \in C(Y)$, and $n \in\langle N(W(P)), C(V(P))\rangle$, with $n$ taking $Y$ to $Y$ in $P$. Thus, the theorem holds for $Y$, and the lemma is proved.

Proof of Theorem 5.1. We complete the proof of this theorem by induction on $|P: X|$. If $X=P$, then $N(X) \leqq N(W(P))$, by the definition of conjugacy functor, and so Theorem 5.1 is true immediately.

Thus, we may assume that $X<P$, and, by induction, that the theorem is true for all subgroups $Y$ of $P$ such that $|P: Y|<|P: X|$.

We show first that there is some conjugate $Y$ of $X$, where $Y$ is a subgroup of $P$, such that the theorem holds for $Y$. By Proposition 2.7, some conjugate $Y$ of $X$ is well-placed in $P$. If $Z(P) \leqq Y$, then Lemma 5.3 shows that the theorem holds for $Y$. If $Z(P) \not Y$, then our induction hypothesis and Lemma 5.7 show that the theorem holds in $Y$. In any case, there is a conjugate $Y$ of $X$ such that the theorem holds in $Y$.

Since the family $F$, described in Definition 2.8 is a conjugation family, and since $(P, N(P)) \in F$, we have the following, using Definition 2.2:

There exist subgroups $X_{1}, \cdots, X_{n}$ of $P$, with $Y=X_{1}$, and $X=X_{n}$, and Sylow $p$-subgroups $Q_{1}, \cdots, Q_{n}$ of $G$, satisfying

(a) $P \cap Q_{i}$ is a well-placed, tame intersection

(b) $X_{i}$ and $X_{i+1}$ are contained in $P \cap Q_{i}$,

(c) if $C_{P}\left(P \cap Q_{i}\right) \geqq P \cap Q_{i}$, then $X_{i}$ and $X_{i+1}$ are conjugate in $C\left(P \cap Q_{i}\right)$, and

(d) if $C_{P}\left(P \cap Q_{i}\right) \leqq P \cap Q_{i}$, then $X_{i}$ and $X_{i+1}$ are conjugate in $N\left(P \cap Q_{i}\right)$.

We show by induction on $j$ that the theorem holds for all $X_{j}$. As $X=X_{n}$, this suffices to prove the theorem.

For $j=1$, we have $X_{1}=Y$, and we have chosen $Y$ so that the 
theorem holds in $Y$. Now suppose that the theorem holds in $X_{j}$. If (c) is true for the index $j$, then $X_{j}$ and $X_{j+1}$ are conjugate in $C\left(P \cap Q_{i}\right)$, and, as they lie in $P \cap Q_{i}$, they are equal, and the theorem holds for $X_{j+1}$.

If, on the other hand, (d) holds for the index $j$, then Lemma $\mathbf{5 . 5}$ shows that the theorem holds for $X_{j+1}$, as a conjugate of $X_{j}$ by an element of $N\left(P \cap Q_{i}\right)$.

This induction completes the proof of Theorem 5.1, and so, of our result on control of strong fusion.

6. Factorizations of the focal subgroup. We first need the following result, which says that a factorization of a $p$-solvable group yields a factorization of its focal subgroup.

Lemma 6.1. Let $G$ be a p-solvable group, $P$ a Sylow p-subgroup of $G$, and suppose $G=\langle H, K, L\rangle$, where $P \leqq H$, and $P \leqq K$, and $L$ is a normal $p^{\prime}$-subgroup of $G$. Then $P \cap G^{\prime}=\left(P \cap H^{\prime}\right) \cdot\left(P \cap K^{\prime}\right)$.

Before we turn to the proof of Lemma 6.1, we prove a preliminary lemma.

Lemma 6.2. If $P$ is a Sylow p-subgroup of the group $G$, and $M$ is a subgroup of $G$ with $P \leqq M$, and $N$ is a normal $p^{\prime}$-subgroup of $G$, then $P N \cap M^{\prime} N=\left(P \cap M^{\prime}\right) N$.

Proof. Call $E=P N \cap M^{\prime} N$. Then $E \leqq P N$, and $N \leqq E$, so, by the Dedekind identity, $E=(P \cap E) N$. Also, $P \cap E=P \cap P N \cap M^{\prime} N=$ $P \cap M^{\prime} N$, so $E=\left(P \cap M^{\prime} N\right) N$. Since $P \cap M^{\prime} N$ is a $p$-group, and $N$ is a $p^{\prime}$-group, this factorization of $E$ shows that $P \cap M^{\prime} N$ is a Sylow $p$-subgroup of $E$. Also, since $M^{\prime}$ is a normal subgroup of $M$ and $P \leqq M$, we have that $P \cap M^{\prime}$ is a Sylow $p$-subgroup of $M^{\prime}$, and hence also of $M^{\prime} N$. Since $P \cap M^{\prime} \leqq E$, and $E \leqq M^{\prime} N$, we have $P \cap M^{\prime}$ a Sylow $p$-subgroup of $E$ as well. But $P \cap M^{\prime} \leqq P \cap M^{\prime} N$, and each of these is a Sylow $p$-subgroup of $E$. Hence, $P \cap M^{\prime}=P \cap M^{\prime} N$, and so $E=\left(P \cap M^{\prime}\right) N$, as required.

Proof of Lemma. 6.1. We proceed by induction on the order of $G$. Thus, we assume that the lemma is true of any group whose order is less than $|G|$.

Let $N$ be a minimal normal subgroup of $G$. Define $\bar{G}=G / N$, $\bar{P}=P N / N, \bar{H}=H N / N, \bar{K}=K N / N$ and $\bar{L}=L N / N$. We claim that these homomorphic images satisfy the hypotheses of the lemma. By elementary properties of homomorphic images, $\bar{G}$ is a $p$-solvable group, $\bar{G}=\langle\bar{H}, \bar{K}, \bar{L}\rangle$, and all the hypotheses of our lemma are satisfied. As 
$|\bar{G}|<|G|$, our induction hypothesis shows that the conclusion of the lemma holds for $\bar{G}$; that is, that $\bar{P} \cap \bar{G}^{\prime}=\left(\bar{P} \cap \bar{H}^{\prime}\right) \cdot\left(\bar{P} \cap K^{\prime}\right)$.

If we consider the corresponding equation for the pre-images in $G$ of these subgroups of $\bar{G}$, using the fact that $\bar{G}^{\prime}=(G / N)^{\prime}=G^{\prime} N / N$, we get

$$
P N \cap G^{\prime} N=\left(P N \cap H^{\prime} N\right) \cdot\left(P N \cap K^{\prime} N\right) \cdot
$$

Define $F=P N \cap G^{\prime} N$. Now $N$ is a minimal normal subgroup of the $p$-solvable group $G$, so $N$ is either a $p$-group or a $p^{\prime}$-group. We consider each of these cases separately.

Case 1. $N$ is a $p^{\prime}$-group. Using, in turn, $G$, then $H$, and then $K$ for $M$ in Lemma 6.2, we have $P N \cap G^{\prime} N=\left(P \cap G^{\prime}\right) N$, and $P N \cap$ $H^{\prime} N=\left(P \cap H^{\prime}\right) N$, and $P N \cap K^{\prime} N=\left(P \cap K^{\prime}\right) N$. Using these equations to rewrite $(6.3)$, we get

$$
F=\left(P \cap G^{\prime}\right) N \text { and } F=\left(P \cap H^{\prime}\right) \cdot\left(P \cap K^{\prime}\right) N .
$$

The first of these equations shows that $P \cap G^{\prime}$ is a Sylow $p$-subgroup of $F$, and the second that $\left(P \cap H^{\prime}\right) \cdot\left(P \cap K^{\prime}\right)$ is a Sylow $p$-subgroup of $F$. But as $\left(P \cap H^{\prime}\right) \cdot\left(P \cap K^{\prime}\right) \leqq P \cap G^{\prime}$, we must have $P \cap$ $G^{\prime}=\left(P \cap H^{\prime}\right) \cdot\left(P \cap K^{\prime}\right)$ as required.

Case 2. $N$ is a $p$-group. As $N$ is a normal $p$-subgroup of $G$, we have $N \leqq P$, so $P N=P$. Thus, (6.3) becomes

$$
F=P \cap G^{\prime} N=\left(P \cap H^{\prime} N\right) \cdot\left(P \cap K^{\prime} N\right) \text {. }
$$

Now, we have $P \cap G^{\prime} N \leqq G^{\prime} N$ and $N \leqq P \cap G^{\prime} N$, so by the Dedekind identity, $P \cap G^{\prime} N=\left(P \cap G^{\prime} N \cap G^{\prime}\right) N$. But $P \cap G^{\prime} N \cap G^{\prime}=$ $P \cap G^{\prime}$, so $P \cap G^{\prime} N=\left(P \cap G^{\prime}\right) N$. Similarly, $P \cap H^{\prime} N=\left(P \cap H^{\prime}\right) N$ and $\left.P \cap K^{\prime} N\right)=\left(P \cap K^{\prime}\right) N$. Thus, the equation above becomes

$$
F=\left(P \cap G^{\prime}\right) N=\left(P \cap H^{\prime}\right) \cdot\left(P \cap K^{\prime}\right) N .
$$

Now $N$ is either an eccentric or a central factor of $G$; that is either $[N, G]>1$ or $[N, G]=1$.

If $[N, G]>1$ then, as $N$ is a normal subgroup of $G,[N, G]$ is a normal subgroup of $G$, and $[N, G] \leqq N$, and so, by the minimality of $N$, we have $[N, G]=N$. We claim that in this case $N \leqq$ $\left(P \cap H^{\prime}\right) \cdot\left(P \cap K^{\prime}\right)$.

Let $n \in N$. Then, as $N=[N, G]$, there exist $m \in N$ and $g \in G$ satisfying $n=[m, g]$. As $G=\langle H, K, L\rangle$, we may write $g=g_{1} g_{2} \cdots g_{n}$, with each $g_{i} \in H, K$, or $L$.

We show by induction that any such commutator $\left[m, g_{1} \cdots g_{k}\right] \epsilon$ $\left(P \cap H^{\prime}\right)\left(P \cap K^{\prime}\right)$, where each $g_{i} \in H, K$, or $L$. This will suffice to 
establish the claim above.

For $i=1$, we have $\left[m, g_{1}\right]$. If $g_{1} \in H$ or $K$, then $\left[m, g_{1}\right] \in N$, since $N$ is normal, and $\left[m, g_{1}\right] \in H^{\prime}$, or $K^{\prime}$ since $m \in N \leqq P \leqq H \cap K$, so [ $\left.m, g_{1}\right] \in N \cap K^{\prime} \leqq P \cap K^{\prime}$. If $g_{1} \in L$, then $\left[m, g_{1}\right] \in N \cap L$, as each of these subgroups in normal; but they are of relatively prime order, so $\left[m, g_{1}\right] \in N \cap L=1$. Thus in any case $\left[m, g_{1}\right] \in\left(P \cap H^{\prime}\right)\left(P \cap K^{\prime}\right)$.

For $i=k$, assume the result holds for $i=k-1$. Call $h=g_{1} \cdots g_{k-1}$. Then $\left[m, g_{1} \cdots g_{k}\right]=\left[m, h g_{k}\right]=\left[m, g_{k}\right][m, h]\left[[m, h], g_{k}\right]$. As in the case above $\left[m, g_{k}\right] \in\left(P \cap H^{\prime}\right)\left(P \cap K^{\prime}\right)$, and, since $[m, h] \in N$, we have

$$
\left[[m, h], g_{k}\right] \in\left(P \cap H^{\prime}\right)\left(P \cap K^{\prime}\right)
$$

for the same reasons. From our induction hypothesis,

$$
[m, h] \in\left(P \cap H^{\prime}\right)\left(P \cap K^{\prime}\right) \text {, so }\left[m, g_{1}, \cdots, g_{k}\right] \in\left(P \cap H^{\prime}\right)\left(P \cap K^{\prime}\right) \text {. }
$$

This completes the induction, and shows that when $[N, G]>1$, we have $N \leqq\left(P \cap H^{\prime}\right)\left(P \cap K^{\prime}\right)$. Since $N \leqq P \cap G^{\prime}$ as well, (6.3) becomes $P \cap G^{\prime}=\left(P \cap H^{\prime}\right)\left(P \cap K^{\prime}\right)$, as required.

The remaining case is $[N, G]=1$; that is, $N \leqq Z(G)$. From (6.3), we can calculate

$$
\left|P \cap G^{\prime}\right|=\frac{|F| \cdot\left|P \cap G^{\prime} \cap N\right|}{|N|}
$$

and

$$
\left|\left(P \cap H^{\prime}\right) \cdot\left(P \cap K^{\prime}\right)\right|=\frac{|F| \cdot\left|\left(P \cap H^{\prime}\right) \cdot\left(P \cap K^{\prime}\right) \cap N\right|}{|N|}
$$

Now, to show that $P \cap G^{\prime}=\left(P \cap H^{\prime}\right) \cdot\left(P \cap K^{\prime}\right)$, it suffices to show that their orders are equal, and for this, it is clear from the equations above, it suffices to show that, $P \cap G^{\prime} \cap N=\left(P \cap H^{\prime}\right) \cdot\left(P \cap K^{\prime}\right) \cap N$. Certainly, $\left(P \cap H^{\prime}\right) \cdot\left(P \cap K^{\prime}\right) \cap N \leqq P \cap G^{\prime} \cap N$.

A transfer theorem ([5], page 416) states that $P \cap G^{\prime} \cap Z(G) \leqq P^{\prime}$. But $N \leqq Z(G)$, so $P \cap G^{\prime} \cap N \leqq P^{\prime}$, and $P \leqq H$; we have $P^{\prime} \leqq P \cap H^{\prime}$. Thus, $P \cap G^{\prime} \cap N \leqq P \cap H^{\prime}$, and so $P \cap G^{\prime} \cap N=\left(P \cap H^{\prime}\right)\left(P \cap K^{\prime}\right) \cap N$, as required. This completes the proof of Lemma 6.1.

We can immediately apply this result to obtain a factorization of the focal subgroup from Theorem 1.8 .

THEOREM 6.4. Let $G$ be a group, $P$ a Sylow p-subgroup of $G$, and $W$ a conjugacy functor satisfying $Z(H) \leqq W(H)$ for all $H \in \mathscr{H}$, and $V$ a central functor. Assume that for every local subgroup $L \in \mathscr{L}$, $L$ is p-solvable, and there is a Sylow p-subgroup $Q$ of $L$ such that $L=\left\langle N(W(Q)), C(V(Q)), O_{p}(L)\right\rangle$. Then 


$$
P \cap G^{\prime}=\left(P \cap N(W(Q))^{\prime}\right)\left(P \cap C(V(Q))^{\prime}\right)
$$

Proof. The theorem follows directly from Lemma 6.1 and Theorem 1.8 (1), our result on local control of transfer.

We now quote several theorems which give conditions under which a group factorizes.

Definition 6.5. A group $H$ is involved in a group $G$ if there are subgroups $K$ and $L$ of $G$, with $K$ a normal subgroup of $L$, such that $H$ is isomorphic to $L / K$; that is, if $H$ is a homomorphic image of some subgroup of $G$.

THEOREM 6.6. (Thompson [6]). Let $G$ be a p-solvable group, and $P$ a Sylow p-subgroup of $G$, and suppose one of the following holds:

(a) $p \geqq 5$

(b) $p=3$ and $S L(2,3)$ is not involved in $G$.

(c) $p=2$ and $S L(2,2)$ is not involved in $G$. Then $G=N_{G}(J(P)) \cdot C_{G}(Z(P)) \cdot O_{p^{\prime}}(G)$.

THEOREM 6.7. (Glauberman [3], p. 19). Let $T$ be a Sylow 2subgroup of $G$, and $C\left(O_{2}(G)\right) \subseteq O_{2}(G)$, and either

(a) $|N(H) / C(H)|<6$ for every four-group $H$ in $Z\left(O_{2}(G)\right)$, or

(b) the symmetric group on four letters is not involved in $G$. Then $G=\langle N(J(T)), C(Z(T))\rangle$.

Theorem 6.8. (Goldschmidt [4]). Suppose $P$ is a Sylow p-subgroup of $G$, and $\left(\widetilde{\mho}^{1} Z(P)\right) \subseteq O_{p}(G)$. Then $G=N(J(P)) \cdot C\left(\widetilde{\mho}^{1}(Z(P))\right)$.

We can now combine any of these three results with Theorem 6.4, and obtain conditions on the local subgroups which imply that the focal subgroup factorizes.

THEOREM 6.9. Assume that every local subgroup $L \in \mathscr{L}$ is $p$ solvable, and satisfies the hypotheses of

(a) Theorem 6.6.

(b) Theorem 6.7, for $p=2$.

(c) Theorem 6.8.

Then, respectively,

(a) and (b) $P \cap G^{\prime}=\left(P \cap N(J(P))^{\prime}\right)\left(P \cap C(Z(P))^{\prime}\right)$, or

(c) $P \cap G^{\prime}=\left(P \cap N(J(P))^{\prime}\right)\left(P \cap C\left(\widetilde{O}^{1}(Z(P))^{\prime}\right)\right.$.

Proof. Apply Theorem 6.4, with $W(H)=J(H)$, and, in the first two cases, $V(H)=Z(H)$, and $V(H)=\mho^{1}(Z(H))$ in the third case. 


\section{REFERENCES}

1. J. L. Alperin, Sylow intersections and fusion, J. Algebra, 6 (1967), 222-241.

2. _ـ and D. Gorenstein, Transfer and fusion in finite groups, J. Algebra, 6 (1967), 242-255.

3. G. Glauberman, Weakly closed elements of Sylow groups, Math. Z., 107 (1968), 1-28.

4. D. M. Goldschmidt, Sylow 2-subgroups with non-elementary centers, Proc. of Symposia in Pure Math., 21 (1971), 53-56.

5. B. Huppert, Endliche Gruppen I, Springer-Verlag, Berlin, 1967.

6. J. G. Thompson, Factorizations of p-solvable groups, Pacific J. Math., 16 (1966), 371-372.

Received November 17, 1971 and in revised form July 17, 1972. This paper is a revision of a part of the author's doctoral thesis at the University of Chicago, prepared under the supervision of Professor J. L. Alperin. The author wishes to thank Professor Alperin for his help and encouragement.

BUCKNELL UNIVERSITY 


\section{PACIFIC JOURNAL OF MATHEMATICS}

EDITORS

\author{
H. SAMELSON \\ Stanford University \\ Stanford, California 94305 \\ C. R. HOBBY \\ University of Washington \\ Seattle, Washington 98105
}

\author{
J. DUGUNDJI \\ Department of Mathematics \\ University of Southern California \\ Los Angeles, California 90007 \\ RICHARD ARENS \\ University of California \\ Los Angeles, California 90024
}

\section{ASSOCIATE EDITORS}

E. F. BECKENBACH

B. H. NeUManN

F. WOLF

K. YosHIDA

\section{SUPPORTING INSTITUTIONS}

UNIVERSITY OF BRITISH COLUMBIA

CALIFORNIA INSTITUTE OF TECHNOLOGY

UNIVERSITY OF CALIFORNIA

MONTANA STATE UNIVERSITY

UNIVERSITY OF NEVADA

NEW MEXICO STATE UNIVERSITY

OREGON STATE UNIVERSITY

UNIVERSITY OF OREGON

OSAKA UNIVERSITY
UNIVERSITY OF SOUTHERN CALIFORNIA

STANFORD UNIVERSITY

UNIVERSITY OF TOKYO

UNIVERSITY OF UTAH

WASHINGTON STATE UNIVERSITY UNIVERSITY OF WASHINGTON

AMERICAN MATHEMATICAL SOCIETY NAVAL WEAPONS CENTER 


\section{Pacific Journal of Mathematics}

\section{Vol. 45, No. $1 \quad$ September, 1973}

William George Bade, Complementation problems for the Baire classes .......... 1

Ian Douglas Brown, Representation of finitely generated nilpotent groups ........ 13

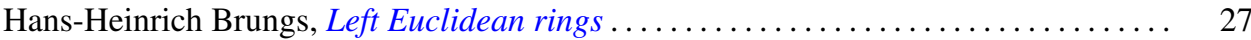

Victor P. Camillo and John Cozzens, A theorem on Noetherian hereditary rings ..... 35

James Cecil Cantrell, Codimension one embeddings of manifolds with locally flat

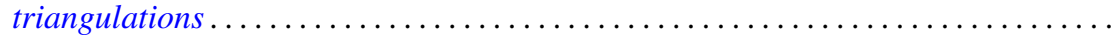

L. Carlitz, Enumeration of up-down permutations by number of rises . . . . . . . . . .

Thomas Ashland Chapman, Surgery and handle straightening in Hilbert cube

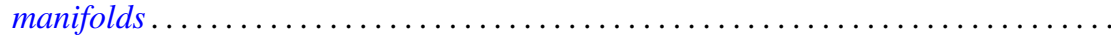

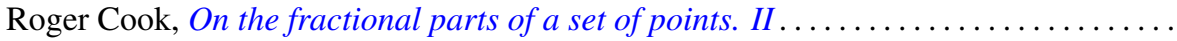

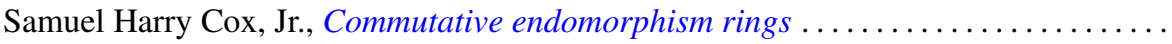

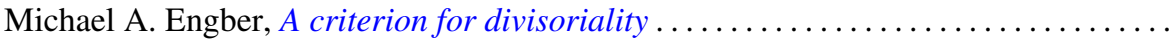

Carl Clifton Faith, When are proper cyclics injective . . . . . . . . . . . . . . 97

David Finkel, Local control and factorization of the focal subgroup . . . . . . . . . 113

Theodore William Gamelin and John Brady Garnett, Bounded approximation by

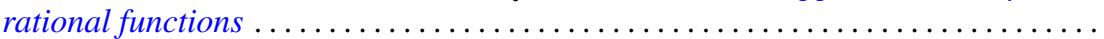

Kazimierz Goebel, On the minimal displacement of points under Lipschitzian

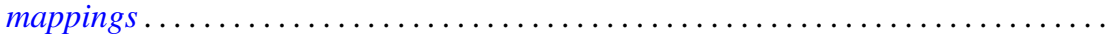

Frederick Paul Greenleaf and Martin Allen Moskowitz, Cyclic vectors for representations associated with positive definite measures: nonseparable

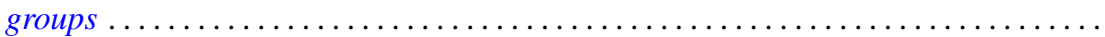

Thomas Guy Hallam and Nelson Onuchic, Asymptotic relations between perturbed linear systems of ordinary differential equations .

David Kent Harrison and Hoyt D. Warner, Infinite primes of fields and completions. .

James Michael Hornell, Divisorial complete intersections . ......

Jan W. Jaworowski, Equivariant extensions of maps ..............

John Jobe, Dendrites, dimension, and the inverse arc function .. .

Gerald William Johnson and David Lee Skoug, Feynman integrals of non-factorable

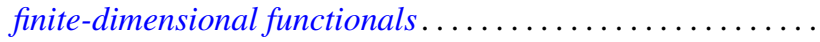

Dong S. Kim, A boundary for the algebras of bounded holomorphic functions ...... 269

Abel Klein, Renormalized products of the generalized free field and its derivatives ... 275

Joseph Michael Lambert, Simultaneous approximation and interpolation in $L_{1}$ and

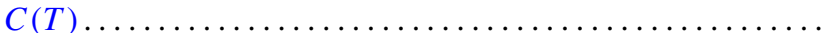

Kelly Denis McKennon, Multipliers of type $(p, p)$ and multipliers of the group

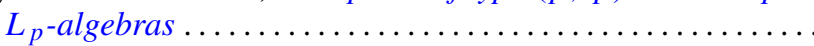

William Charles Nemitz and Thomas Paul Whaley, Varieties of implicative

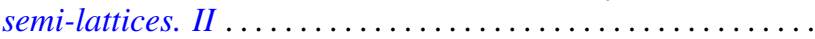

Donald Steven Passman, Some isolated subsets of infinite solvable

Norma Mary Piacun and Li Pi Su, Wallman compactifications on E-completely

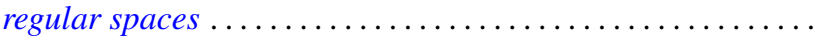

Jack Ray Porter and Charles I. Votaw, $S(\alpha)$ spaces and regular Hausdorff extensions....

Gary Sampson, Two-sided $L_{p}$ estimates of convolution transforms .

Ralph Edwin Showalter, Equations with operators forming a rig
Raymond Earl Smithson, Fixed points in partially ordered sets .

Victor Snaith and John James Ucci, Three remarks on symmetric products and

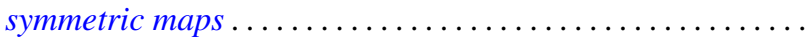

